\title{
THE ADJOINT REPRESENTATION OF GROUP ALGEBRAS AND ENVELOPING ALGEBRAS
}

\author{
D. S. PASSAAN*
}

Dedicaled to the nemony of Pere Menal

\begin{abstract}
In this paper we study the llopr adjoint action of group algebras and enveloping algebras. We are pari,icularly concorned with determining when these representations are faithful. Delta methods allow us to reduce the problem to certain better behaved subalgebras. Nevertheless, the problem remains open in the finito group and frite-dimensional Lic algebra cases.
\end{abstract}

\section{Group Algebras}

Suppose $\mathcal{H}$ is a Hopf algebra with comultiplication $\mathcal{H} \rightarrow \mathcal{H} \otimes \mathcal{H}$ given by $h \mapsto \sum_{(h)} h_{1} \otimes h_{2}$ and with antipode $S: \mathcal{H} \rightarrow \mathcal{H}$. Then the left adjoint action of $\mathcal{H}$ on $\mathcal{H}$ is defined by $h_{h} \cdot x=\sum_{(h)} h_{1} x S\left(h_{2}\right)$ for all $h, x \in \mathcal{H}$. In this way, $\mathcal{H}$ becomes a left $\mathcal{H}$-module and therefore the kernel of this action

$$
\mathcal{I}(\mathcal{H})=\{h \in \mathcal{H} \mid h \cdot \mathcal{H}=0\}
$$

is a two-sided ideal of $\mathcal{H}$. It is of interest to determine when $\mathcal{H}$ acts faithfully, that is when $\mathcal{I}(\mathcal{H})=0$. In this paper, we will be concerned with the Hopf algebras $\mathcal{H}=K[G$; and $\mathcal{H}=U(L)$.

If $\mathcal{H}=K[G]$ is the group algebra of $G$ over the field $K$, then the comultiplication and antipode are given by $g \mapsto g \otimes g$ and $S(g)=g^{-1}$, respectively, for all $g \in C$. In particular, the left adjoint action is just: $g \cdot a=g x g^{-1}$, ordinary conjugation by the group elenent $g$. It follows that if $z$ is a central element of $G$, then $z-1 \in \mathcal{I}(G)$. Here, for convenience, we have abbreviated $\mathcal{I}(K[G])$ by $\mathcal{I}(G)$. Thus $\mathbb{Z}(G) \neq 1$ implies

*Rescarch supported in part by NSF Grant DMS-8900405. 
that $\mathcal{I}(G) \neq 0$. As we will see, the converse of this staternent is definitely not true. Nevertheless, be begin by proving that $\mathcal{I}(G)$ is controlled by the f.c. center of $G$, namely

$$
\Delta=\Delta(G)=\left\{x \in G|| G: \mathbb{C}_{G}(x) \mid<\infty\right\} .
$$

Theorem 1.1. If $\mathcal{I}(G)$ is the kernel of the adjoint representation of $K[G]$, then

$$
\mathcal{I}(G)=(\mathcal{I}(G) \cap K[\Delta]) K[G]=K[G](\mathcal{I}(G) \cap K[\Delta]) .
$$

In particular, if $\Delta(G)=1$, then $\mathcal{I}(G)=0$.

Proof: As usual, let $\theta: K[G] \rightarrow K[\Delta]$ denote the $K$-lincar projection map defined on $G$ by $\theta(g)=g$ if $g \in \Delta$ and $\theta(g)=0$ otherwise. If $\alpha=\sum_{g \in G} a_{g} g \in \mathcal{I}(G)$, then for all $\beta \in K[G]$ we have

$$
0=\alpha \cdot \beta=\sum_{g \in G} a_{g} g \beta g^{-1}
$$

Thus $[\mathbf{P}$, Lemma 4.2.5] applied to this linear identity yields

$$
0=\sum_{g \in G} \theta\left(a_{g} g\right) \beta g^{-1}=\sum_{g \in \Delta} a_{g} g \beta g^{-1}=\theta(\alpha) \cdot \beta
$$

and hence $\theta(\alpha) \in \mathcal{I}(G)$. [P, Lemma 1.1.5] now implies that $\Delta(G)$ controls $\mathcal{I}(G)$, that is

$$
\mathcal{I}(G)=(\mathcal{I}(G) \cap K[\Delta]) K[G]=K[G](\mathcal{I}(G) \cap K[\Delta]) .
$$

Finally, if $\Delta=1$, then certainly $\mathcal{I}(G) \cap K[\Delta]=\mathcal{I}(G) \cap K=0$ and $\mathcal{I}(G)=0$.

To proceed further we require a better understanding of $\mathcal{L}(G) \cap K[\Delta]$. As we see below, there is an easy description of this ideal, and of $\mathcal{I}(G)$ for that matter, based on the fact that the adjoint representation is a permutation representation. Suppose $G$ permutes a set $\Omega$ and consider the $K$-vector space $V=K \Omega$ having $\Omega$ as a basis. Then $V$ is naturally a left $K[G]$-module, the permutation module associated with $(G, \Omega)$. Furthermore, if $x \in \Omega$, then the annihilator of $x$ in $K[G]$ is clearly equal to $K[G] \omega\left(G_{x}\right)$ where $G_{x}$ is the stabilizer of $x$ in $G$ and where $\omega\left(G_{x}\right)$ denotes the augmentation ideal of $K\left[G_{*}\right]$. In particular, since the adjoint representation of $K[G]$ corresponds to the conjugation permutation module with $\Omega=G$ and with $G_{x}=\mathbb{C}_{G}(x)$, the preceding considerations applied to $G$ and to $\triangle$ yield 
Lemma 1.2. With the above notation

$$
\mathcal{I}(G)=\bigcap_{x \in G} K[G] \omega\left(\mathbb{C}_{G}(x)\right)
$$

and

$$
\mathcal{I}(G) \cap K[\Delta]=\bigcap_{x \in G} K[\Delta] \omega\left(\mathbb{C}_{\Delta}(x)\right)
$$

It is a simple consequence of this result that if $F$ is a field extension of $K$, then $\mathcal{I}(F[G])=F \otimes \mathcal{I}(K[G])$. Thus the vanishing of $\mathcal{I}(G)$ depends only upon $G$ and the characteristic of the field. Sone interesting eximples are as follows.

Lemma 1.3. i. Suppose $G$ has a nonidentity normal torsion-free abelian subgroup $A$ with $|G / A|<\infty$ and $\mathbb{C}_{G}(A)=A$. Then $\mathcal{I}(G)=0$ if and only if some element of $G / A$ acts without fixed points on $A$.

i. Let $p$ and $q$ be primes with $p \mid q-1$ and form the group $G=Q \times P$. Lere $P$ is an elementary abelian $p$-group of order $p^{2}, Q$ is an elementary abelian q-group of order $q^{p+1}$ and each subyroup of $P$ of order $p$ is the kernel of the action of $P$ on a cyclic factor of $Q$. Then $\mathbb{Z}(G)=1$, but $\mathcal{I}(G) \neq 0$.

Proof: (i) Since $A$ is self-centralizing, $G / A$ acts faithfully on $A$. Furthermore, since $G / A$ is finite and $A$ is torsion free, it follows that $A=$ $\Delta(G)$ and that $K[A]$ is a commutative domain. Now the preceding fermm inplies that

$$
\mathcal{I}(G) \cap K[\Delta]=\bigcap_{g \in C / A} K[A] \omega\left(\mathbb{C}_{A}(g)\right)
$$

In particular, if some $\mathbb{C}_{A}(g)=1$, then $\mathcal{I}(G) \cap K[\Delta]=0$ and $\mathcal{I}(G)=0$ by Theorem 1.1. On the other hand, if all $\mathbb{C}_{A}(g) \neq 1$, then each of the finitely many ideals $K[A] \omega\left(\mathbb{C}_{A}(g)\right)$ is nonzero. Thus, since $K[A]$ is a domain, it follows that $\mathcal{I}(G) \cap K[\Delta] \neq 0$ and hence that $\mathcal{I}(G) \neq 0$.

(ii) Write $Q=Q_{0} \times Q_{1} \times \cdots \times Q_{p}$ where each $Q_{i}$ is cyclic of order q. Since $p \mid q-1$ and $P$ has precisely $p+1$ subgroups of order $p$, say $P_{0}, P_{1}, \ldots, P_{p}$, we can certainly define the action of $P$ on $Q$ so that the cyclic group $P / P_{i}$ acts faithfully on $Q_{i}$. It then follows easily that for each $g \in G=Q \rtimes P$, there exists a subscript $i$ with $\mathbb{C}_{C}(g) \supseteq Q_{i}$. Thus the preceding lemma implics that $\mathcal{I}(G) \supseteq \prod_{i=0}^{p} \omega\left(Q_{i}\right) \neq 0$. Since $\mathbb{Z}(G)=1$, the result follows.

There are of coluse numerous variants of the construction in (ii). Lemma 1.2 also yields a second characterization of $\mathcal{I}(G)$. 
Lemma 1.4. For each $x \in G$, let $\pi_{x}: K[G] \rightarrow K\left[\mathbb{C}_{G}(x)\right]$ denote the natural projection map. Then $\mathcal{I}(G)$ is the largest two-sided ideal $I$ of $K[G]$ with $\pi_{x}(l) \subseteq \omega\left(\mathbb{C}_{G}(x)\right)$ for all $x \in G$. In particular, $\mathcal{I}(G)$ is also the kernel of the right adjoint representation of $K[G]$.

Proof: For any $x \in G$ we have $\mathcal{I}(G) \subseteq K[G] \omega\left(\mathbb{C}_{G}(x)\right)$ and hence $\pi_{x}(\mathcal{I}(G)) \subseteq \omega\left(\mathbb{C}_{G}(x)\right)$. Conversely, suppose that $I$ is a two-sided ideal of $K[G]$ with $\pi_{x}(I) \subseteq \omega\left(\mathbb{C}_{G}(x)\right)$ for all $x \in G$. Since $I$ is a left ideal, it follows from [P. Lemma 1.1.3] that $I \subseteq K[G] \omega\left(\mathbb{C}_{G}(x)\right)$ for all such $x$ and therefore that $I \subseteq I(G)$ by Lemma 1.2. Finally, this description of $\mathcal{I}(G)$ is right-left symmetric, and thus we conclude that $\mathcal{I}(G)$ is also the kernel of the right adjoint representation.

Now Theorem 1.1 reduces the study of $\mathcal{I}(G)$ to $K[\Delta]$ and we know that $\Delta(G)$ contains all the finite normal subgroups of $G$. Thus an appropriate first step in studying $K[\Delta]$ is to consider the case where $G$ is finite.

We assume throughout the remainder of this section that $G$ is a finite group. If $H$ is a subgroup of $G$, define

$$
\bar{H}=\sum_{h \in H} h \in K[H] \subseteq K[G] .
$$

Notice that $\overline{g H g^{-1}}=g \bar{H} g^{-1}$ and that if $H_{1} \subseteq H_{2}$, then $\overline{H_{1}}$ divides $\overline{H_{2}}$. Furthermore, let $\mathcal{A}(G)$ denote the two-sided ideal of $K[G]$ generated by the elements $\overline{C_{G}(x)}$ for all $x \in G$.

Lemma 1.5. If $G$ is a finite group, then

$$
\operatorname{ann}_{K[G]} \mathcal{I}(G)=\mathcal{A}(G)
$$

and

$$
\operatorname{ann}_{K\lfloor G j} \mathcal{A}(G)=\mathcal{I}(G) \text {. }
$$

In particular, $\mathcal{I}(G)=0$ if and only if $1 \in \mathcal{A}(G)$.

Proof: Set $\mathcal{A}^{\prime}(G)=\sum_{x \in G} \overline{\mathrm{C}_{G}(x)} K[G]$ so that $\mathcal{A}^{\prime}(G)$ is a right ideal of $K[G]$ contained in $\mathcal{A}(G)$. Since $\mathbb{C}_{G}\left(x^{g}\right)=\mathbb{C}_{C}(x)^{g}$, it follows that $\mathcal{A}^{\prime}(G)$ is a $G$-stable right ideal and therefore a two-sided ideal of $K[G]$. Thus clearly $\mathcal{A}^{\prime}(G)=\mathcal{A}(G)$. Now if $H$ is a subgroup of $G$, then we know that l.ann $\bar{H}=K[G] \omega(H)$. Thus, the above description of $\mathcal{A}(G)$ implies that

$$
\operatorname{lann} \mathcal{A}(G)=\bigcap_{x \in G} K[G] \omega\left(\mathbb{C}_{G}(x)\right)=\mathcal{I}(G)
$$


by Lemna 1.2. Furthermore, since $K[G]$ is a Frobenius algebra, we conclude that.

$$
\operatorname{ranu} \mathcal{I}(G)=\mathrm{r} \cdot \operatorname{arn}(\operatorname{lann} \mathcal{A}(G))=\mathcal{A}(G) \text {. }
$$

Finally, since $\mathcal{A}(G)$ is symmetrically defined: we can use the right-left symmetry of $\mathcal{I}(G)$ given by the previous lemma to reverse the roles of the right and left annihilators in the above formulas. Thus

$$
\mathcal{I}(G)=\operatorname{lann} \mathcal{A}(G)=\operatorname{rann} \mathcal{A}(G)
$$

and similariy

$$
\mathcal{A}(G)=\operatorname{rann} \mathcal{I}(G)=1 \cdot \operatorname{ann} \mathcal{I}(G)
$$

as required. Alternately, we can use the fact that $K[G]$ is a symmetric algebra and that in such algebras right and left annihilators of two-sided ideals are equal.

For convenicnce, we say that a subset $X$ of $G$ "spans 1 " if the element 1 is contained in the two-sided ideal of $K[G]$ generated by the elements $\overline{\mathbb{C}_{G}(x)}$ for all $x \in X$. Obviously, if some such subset spans 1, then $1 \in \mathcal{A}(G)$ and $\mathcal{I}(G)=0$. Our next examples are Frobenius groups.

Lemma 1.6. i. Let $K$ be a field of characteristic $p>2$ and let $G$ be the dinedrul group of order $2 p$. Then $\mathcal{I}(G) \neq 0$ even though $\mathbb{Z}(G)=1$.

ii. Let $K$ be a field of characteristic 0 and let $G=N C$ be a Frobenius group with kernel $N$ and complement $C$. If $1 \neq x \in N$ and $1 \neq y \in C$, then $\{x, y\}$ spans 1 and herce $\mathcal{I}(G)=0$.

Proof: (i) Let $P$ be the subgronp of $G$ of order $p$ and let $C_{1}, C_{2}, \ldots, C_{p}$ be the subgroups of $G$ of order 2 . Then these subgroups and $G$ itself are the centralizers of elements of $G$. Now let $\lambda: K[G] \rightarrow K$ be the $K$-homomorphism defined by $\lambda(g)=1$ if $g \in P$ and $\lambda(g)=-1$ if $g \in$ $G \backslash P$. Since $K$ has characteristic $p$, it follows that $\bar{P}, \overline{C_{i}}$ and $\bar{G}$ are all contained in the kernel of $\lambda$. Thus $A(G) \neq K[G]$ and $I(G) \neq 0$. Indeed, $0 \neq \bar{G}-2 \bar{P} \in \mathcal{I}(G)$ since this element; is central and annihilates all generators of $\mathcal{A}(G)$.

(ii) Basic properties of $G$ imply that $\mathbb{C}_{G}(x) \subseteq N$ and $\mathbb{C}_{G}(y) \subseteq C$. Therefore $\bar{N}, \bar{G}$, and $\overline{g C g^{-1}}$ are all contained in the ideal $I$ of $K[G]$ generated by $\overline{\mathbb{C}_{G}(x)}$ and $\overline{\mathbb{C}_{C}(y)}$. Finally, if $|N|=n$, then we know that $G$ is the "disjoint" union of $N$ and of the $n$ conjugates $C_{1}, C_{2}, \ldots, C_{72}$ of C. Thus

$$
\eta \bar{I}=\bar{N}+\sum_{1}^{n} \overline{C_{i}}-\bar{C} \in I
$$


and, since $K$ has characteristic 0 , we conclude that $1=\overline{1} \in I$.

Since the dihedral group of order $2 p$ with $p>2$ is a Frobenius group, the preceding lemma shows that the vanishing of $\mathcal{I}(G)$ does indeed depend on the characteristic of the field. For the remainder of this section we will assume that $K$ has characteristic 0 . For the most part, we will be concerned with the symmetric groups Symi ${ }_{t}$. To start with, we have

Lemma 1.7. Let $K$ be a field of characteristic 0 , let $G=\mathrm{Sym}_{n}$ and Let $p$ be an odd prime.

i. Suppose $n \geq p$, let $P$ be the cyclic subgroup of $G$ generated by a p-cycle and let $Q$ be the cyclic subgroup of $G$ generated by a $(p-1)$-cycle. Then

$$
K[G] \bar{P} K[G]+K[G \mid \bar{Q} K[G]=K[G] .
$$

ii. If $n=p$, then the set consisting of any $p$-cycle and any $(p-1)$ cycle spans 1 .

iii. If $n=4$, then the set consisting of any nonidentity 2-element and any non-identity 3 -elernent spans 1 .

Proof: (i)(ii) Assume that $n \geq p$. Let $F=\mathrm{GF}(p)$ and considet the usual group $H$ of linear functions on $F$ described by $\zeta \mapsto a \zeta+b$ for all $a, b \in F$ with $a \neq 0$. 'Then $H=N C$ is a Froberius group with kernel $N$ of order $p$ and complement $C$ of order $p-1$. Since $H$ faithfully permutes the elements of $F$, we can view $H$ as beirg embedded in $\mathrm{Sym}_{p} \subseteq \mathrm{Sym}_{n}=G$. In this embedding, $N$ is generated by a $p$-cycle $x$ and $C$ is generated by a $(p-1)$-cycle $y$. Hence, since $\mathbb{C}_{H}(x)=N$ and $\mathbb{C}_{H}(y)=C$, Lemma 1.6 (ii) implies that

$$
1 \in K[H] \bar{N} K[H]+K[H] \bar{C} K[H] \subseteq K[G] \bar{N} K[G]+K[G] \bar{C} K[G] .
$$

But $P$ is conjugate to $N$ in $G$ and $Q$ is conjugate to $C$ in $C$, so

$$
\text { 1. } \in K[G] \bar{P} K[G]+K[G] \bar{Q} K[G]
$$

and (i) is proved. Finally, if $n=p$, then $P$ is the centralizer in $G$ of a $p$-cycle and $Q$ is the centralizer in $G$ of a $(p-1)$-cycle. "Thus (ii) follows.

(iii) In $G=$ Sym $_{4}$, let $A=$ Alt $_{4}$ and let $N$ denote the Klein four subgroup. Furthermore let $D_{1}, D_{2}$ and $D_{3}$ be the Sylow 2-subgroups of $G$ and let $C_{1}, C_{2}, C_{3}$ and $C_{4}$ be the Sylow 3 -subgroups. Notice that $D_{i} \cap D_{j}=N$ and $C_{i} \cap C_{j}=1$ for all appropriate $i \neq j$. Thus since all elements of $G$ are either 2-elements or 3-elements, we have

$$
\sum_{i} \overline{D_{i}}+\sum_{j} \overline{C_{j}}=\bar{G}+2 \bar{N}+4 \overline{1} .
$$


Similarly, by considering the alternating group $A$, we obtain

$$
\bar{N}+\sum_{j} \overline{C_{j}}=\bar{A}+4 \overline{1} .
$$

'Thus, adding twice the second equation to the first and canceling the $\bar{N}$ terms yields

$$
\sum_{i} \overline{D_{i}}+3 \sum_{j} \overline{C_{j}}=\bar{G}+2 \bar{A}+12 \overline{1} \text {. }
$$

Finally, if $x$ is a nonidentity 2-clement of $G$ and if $y$ is a nonidentity 3-clement, then $\mathbb{C}_{C}(x) \subseteq D_{i}$ and $\mathbb{C}_{C}(y)=C_{j}$ for some $i, j$. Thus the idcal $I$ of $K[G]$ generated by $\overline{C_{G}(x)}$ and $\overline{\mathbb{C}_{G}(y)}$ contains $\overline{D_{i}}$ and all its conjugates as well as $\overline{C_{j}}$ and all its conjugates. Furthermore, since $C_{j} \subseteq A \subseteq G$, we see that $I$ contains $\bar{A}$ and $\bar{G}$. Thus $12 \overline{1} \in I$ and, since $K$ has characteristic 0 , we conclude that $1=\overline{1} \in I$.

It is obvious that, $\mathcal{I}\left(\right.$ Syn $\left._{1}\right)=0$ and that $\mathcal{I}\left(\right.$ Sym $\left._{2}\right)=\omega\left(\right.$ Sym $\left._{2}\right) \neq 0$. Our goal is to show that $\mathcal{I}\left(\mathrm{Sym}_{n}\right)=0$ for all $n \geq 3$ and the proof will proceed by induction on $n$. The following lemma shows how the inductive hypothesis is used. Part (i) is an unpleasant techical formulation which is needed to handle a few small cases.

Lemma 1.8. Let $G=\operatorname{Sym}_{n}$, let $C$ be the cyclic subgroup of $G$ generated by a $k$-cycle $c$ and let $H=\operatorname{Sym}_{r-k}$.

i. Suppose that $X$ is the set of elements of $H$ which do not have a $k$-cycle in their cycle decomposition and assume that $X$ spans 1 in $K[H]$. If $Y$ is the set of elements of $G$ uhich have precisely one $k$-cycle in their decomposition, then

$$
\vec{C} \in \sum_{y \in Y^{Y}} K[G] \overline{\mathbb{C}_{G}(y)} K[G] \subseteq \mathcal{A}(G) .
$$

ii. If $k>n / 2$ and $\mathcal{A}(H)=K[H]$, then $\bar{C} \in \mathcal{A}(G)$.

Proof: (i) Consider the usual embedding of $\operatorname{Sym}_{k} \times \operatorname{Sym}_{n-k}$ in $G=$ Sym $_{n}$ and suppose that $c \in \mathrm{Sym}_{k}$ and that $H=\mathrm{Sym}_{n-k}$. If $x \in X \subseteq H$, then $\mathbb{C}_{G}(c x)=C \times \mathbb{C}_{H}(x)$ since $c$ is a $k$-cycle and since, by hypothesis, $x$ has no $k$-cycle in its decomposition. Thus, since $c x \in Y$, we see that $\bar{C} \overline{\mathbb{C}_{H}(x)}=\overline{\mathbb{C}_{G}(c x)}$ is contained in $I=\sum_{y \in Y} K[G] \overline{\mathbb{C}_{G}(y)} K[G]$. Furthermore, since $\bar{C}$ commutes with $K[H]$, it follows that $I$ contains

$$
\sum_{x \in X} K[H] \bar{C} \overline{\mathbb{C}_{H}(x)} K[H]=\bar{C} \sum_{x \in X} K[H] \overline{\mathbb{C}_{H}(x)} K[H] .
$$


But, $1 \in \sum_{x \in X} K[H] \overline{\mathbb{C}_{H}(x)} K[H]$, by assumption, and therefore $\bar{C} \in I \subseteq$ $\mathcal{A}(G)$ as required.

(ii) If $\mathcal{A}(I)=K[H]$, then obviously $X=H$ spans 1 ir $K[H]$. Furthermore, since $k>n / 2$, we have $k>n-k$ and therefore $X$ consists of the elements of $H$ which do not have a $k$-cycle in their cycle decomposition. The result now follows from (i).

Next we consider two special cases with small values of $n$.

Lemma 1.9. Let $G=\mathrm{Sym}_{n}$.

i. If $n=6$ and if $X$ is the set of all elements of $G$ which are not 6-cycles, then $X$ spans 1 .

ii. If $n=9$, then $A(G)=K[G]$.

Proof: We start with a general observation. Let $\sigma: G \rightarrow\{ \pm 1\}$ be the natural group homomorphism determined by the parity map $\sigma$ and extend this to a $K$-algebra homomorphism $\sigma: K[G] \rightarrow K$. Notice that if $t$ is a transposition in $G$, then $1+t \in \operatorname{Ker}(\sigma)$. Indecd, wo claim that $K[G](1+t) K[G]=\operatorname{Ker}(\sigma)$. To this end, let $I$ denote the left-hand ideal and set $W=\{g \in G \mid g-\sigma(g) \in I\}$. Then $W$ is easily seen to be a normal subgroup of $G$ and, since $t \in W$, we have $W=G$ and hence clearly $I=\operatorname{Ker}(\sigma)$. Define $T$ to be the subgroup of $G$ of order 2 generated by $t$.

(i) Let $n=6$ and continue with the above notation. Furthermore, let $Y$ be the set of elements of $G$ which contain precisely one 2-cycle in their decomposition. If $H=\mathrm{Sym}_{4}$, then we know from Lemma 1.7(iii) that the set $X \subseteq H$ consisting of a 4-cycle and a 3-cycle spans 1. Thus since $t$ is a 2-cycle, Lemma $1.8(\mathrm{i})$ implies that

$$
1+t=\bar{T} \in \sum_{y \in Y} K[G] \overline{\mathbb{C}_{C}(y)} K[G]
$$

and, by the observation of the first paragraph, the preceding ideal contains $\operatorname{Ker}(\sigma)$ and has codimension at most 1 . But if $y=(12)(3456) \in Y$; then $\mathbb{C}_{G}(y) \subseteq$ Alt $_{6}$ and hence $\overline{\mathbb{C}_{G}(y)} \notin \operatorname{Ker}(\sigma)$. With this, we conclude that $Y$ spans 1 and, since $Y$ contains no 6-cycle, this part is proved.

(ii) Similarly, let $n=9$ and set $H=\mathrm{Sym}_{7}$. Then, by Lcmma 1.7 (ii), the set $X \subseteq H$ consisting of a 7 -cycle and a 6 -cycle spans 1 . Again, Lemma 1.8(i) implies that $1+t=\bar{T} \in \mathcal{A}(G)$ and therefore $\mathcal{A}(G) \supseteq$ $\operatorname{Ker}(\sigma)$. But if $y \in G$ is a 9-cycle, then $\mathbb{C}_{G}(y) \subseteq$ Alts and therefore $\overline{\mathbb{C}_{G}(y)} \notin \operatorname{Ker}(\sigma)$. As before, it follows that $\mathcal{A}(G)=K[G]$.

We now use Bertrand's Postulate to handle the remaining symmetric groups. 
Theorem 1.10. If $K$ has characteristic 0 , then $\mathcal{I}\left(\mathrm{Sym}_{n}\right)=0$ for all $n \geq 3$.

Proof: Write $G=$ Sym $_{n}$ and proceed by induction on $n \geq 3$. In view of Lemma 1.5, our goal is to show that $\mathcal{A}(G)=K(G)$. If $n$ is a prime, then this is certainly the case by Lemma 1.7 (ii) and, in particular, this starts the induction when $n=3$. We can now assume that $n$ is not a prime and that $n \geq 4$.

Suppose that there exists a prime number $p$ with $n / 2<p \leq n$ and with $p \neq n-1, n-2,(n+1) / 2$ or $(n+2) / 2$. In this case, since $n \neq p$ by assumption, we see that if $k=p$ or $p-1$, then $n>n-k \geq 3$ and furthermore $k>n / 2$. By induction we know that $1 \in A\left(\operatorname{Sym}_{n-k}\right)$ and therefore Lemma 1.8 (ii) implies that $A(C)$ contains $\bar{P}$ and $\bar{Q}$ where $P$ is generated by a $p$-cycle and $Q$ is generated by a $(p-1)$-cycle. Since $p>n / 2$ is odd, we conclude from Lemma $1.7($ i) that $\mathcal{A}(G)=K[G]$ and hence that $\mathcal{I}(G)=0$.

It remains to show that such a prime $p$ exists except for a few small values of $n$. For any positive real number $\zeta$, let $\pi(\zeta)$ denote the number of primes less than or equal to $\zeta$. Then the set of primes $p$ with $n / 2<p \leq n$. has size $\pi(n)-\pi(n / 2)$. Now according to [RS, Theorem 2, Corollary '3] we have

$$
\pi(2 \zeta)-\pi(\zeta)>3 \zeta / 5 \log \zeta \quad \text { for all } \zeta \geq 20.5
$$

and hence

$$
\pi(n)-\pi(n / 2)>3(20.5) / 5 \log (20.5)>4 \text { for all } n \geq 41 \text {. }
$$

With this, it is simple to check that

$$
\pi(n)-\pi(n / 2) \geq 3 \quad \text { for all } n \geq 17
$$

In particular, if $n \geq 17$ then there are at least three primes $p$ with $n / 2<p \leq n$. On the other hand, we note that $n-1$ and $n-2$ camnot both be prine and that $(n+1) / 2$ and $(n+2) / 2$ camot both be integers. Thus the restriction $p \neq n-1, n-2,(n+1) / 2$ or $(n+2) / 2$ eliminates at most two primes from this segment and we conclude that an appropriate $p$ does indeed exist for $n \geq 17$. Therefore the induction step carries through in this range.

Recall that $n \geq 4$ and that $n$ is not a prime. Furthermore, note that we do not really need $\pi(n)-\pi(n / 2)$ to be $\geq 3$. Rather, we just need the existence of an appropriate prime and clearly $p=11$ works for $n=16,15,14$ and $p=7$ works for $n=10$. This leaves only the special cases $n=4,6,8,9,12$ to be considered and we already have affirmative 
answers for $n=4$ from Lemma 1.7 (iii) and for $n=6,9$ from Lemma 1.9. Thus there are only two integers left.

If $n=12$, let $p=7$. Then it follows from Lemma 1.8 (ii) that $\bar{P} \in \mathcal{A}(G)$ where $P$ is the subgroup of $G$ generated by a 7-cycle. Furthermore, by Lemma 1.9(i), the set of elements of Sym $\mathrm{B}_{6}$ which are not 6-cycles spans 1. Thus Lemma 1.8 (i) with $k=p-1=6$ and $n-k=6$ implies that $\bar{Q} \in \mathcal{A}(C)$ where $Q$ is generated by a 6 -cycle. Lemma 1.7 (i) therefore yields the result in this case. Similarly, if $n=8$ then we let $p=5$ and use the fact, given in Lemma 1.7(iii), that a 2-cycle and a 3-cycle span 1 in $K\left[\mathrm{Sym}_{4}\right]$. This again guarantees that $\mathcal{A}(G)$ contains an appropriate $\bar{P}$ and $\bar{Q}$ and the theorem is proved.

If $K$ is an algebraically closed field of characteristic 0 , then the representation theory of $K \mid G]$ is equivalent, in some sense, to the character theory of the finite group $C$. Thus it is reasonable to translate the problem studied here into character-theoretic language. Since this translation is well known, we will just mention a few basic facts. We follow the notation of [I].

Lemma 1.11. Assume that $K$ is algebraically closed and let $\phi: G \rightarrow$ $K$ denote the character of the adjoint representation of $K[G]$. Then

i. $\phi(g)=\left|\mathbb{C}_{G}(g)\right|$ for all $g \in G$ and

$$
\phi=\sum_{x}\left(1_{\mathfrak{C}_{G}(x)}\right)^{G}
$$

where the sum is over representatives $x$ of the conjugacy classes of $G$.

ii.

$$
\phi=\sum_{\psi \in \operatorname{Irr}(G)} \psi \bar{\psi}
$$

where $\bar{\psi}(g)=\psi\left(g^{-1}\right)$ for all $g \in G$.

Proof: (i) Since $\phi$ is the character of the conjugation permutation representation, it is clear that $\phi(g)=\left|\mathbb{C}_{G}(g)\right|$, the number of elements $x \in G$ which are fixed by $g$. The second formula is also clear since $\phi$ is the sum of the characters which correspond to the action of $G$ on individual conjugacy classes.

(ii) The expression $\phi=\sum_{\psi \in \operatorname{lrr}(G)} \psi \bar{\psi}$ can be deduced from the character orthogonality relations $[\mathrm{I}$, Theorem 2.18$]$ and the fact that $\phi(g)=$ $\left|\mathbb{C}_{G}(g)\right|$. A more natural proof starts by writing $K[G]=\sum_{\psi \in \operatorname{lrr}(G)} M(\psi)$, a direct sum of full matrix rings over $K$, with $M(\psi)$ corresponding to 
the character $\psi$. Then each $M(\psi)$ is a sulmodule of $K[G]$ under the adjoint action and, as is well known, the character of this submodule is $\psi \bar{\psi} \bar{\psi}$.

It is obvious that the adjoint representation of $K[G]$ is faithful if and only if every irreducible character $\chi \in \operatorname{Ir}(C)$ is a constituent of $\phi$. Thus the above and Frobenins reciprocity yield

Lemma 1.12. If $K$ is algebraically closed, then $I(G)=0$ if and only if, for each $\chi \in \operatorname{Irr}(G)$, we have

i. $\chi$ is a constituent of $\left(1_{\mathbb{C}_{C(x)}}\right)^{G}$ for some $x \in G$, or

ii. $1_{\mathbb{C}_{G}(x)}$ is a constituent of $\chi$ restricted to $\mathbb{C}_{G}(x)$ for some $x \in G$, or

iii. $\chi$ is a constituent of $\psi \bar{\psi} \bar{\psi}$ for some $\psi \in \operatorname{Irr}(G)$.

There is obviously a good deal yet to be done on this topic.

\section{Enveloping Algebras}

Now let $L$ be a Lie algebra over the field $K$ and let $U(L)$ denote its miversal enveloping algebra. Then $\mathcal{H}=U(L)$ is a Hopf algebra with comultiplication and antipode detemined by $\ell \mapsto \ell \otimes 1+1 \otimes \ell$ and $S(\ell)=-\ell$, respectively, for all $\ell \in L$. Of course, $1 \mapsto 1 \otimes 1$ and $S(1)=1$. It follows that the lopf left adjoint action of $U(L)$ on itself is determined by $\ell \cdot x=\ell x-x \ell$ for all $\ell \in L$ and $x \in U(L)$. In other words, $\ell \cdot x=[\ell, x]=($ ad $\ell) x$. Again we are concerned with the kernel of the adjoint representation, namely

$$
\mathcal{I}(L)=\mathcal{I}(U(L))=\{\alpha \in U(L) \mid \alpha \cdot U(L)=0\}
$$

It is clear that $\mathcal{I}(L) \cap L=\mathbb{Z}(L)$ and therefore, as with groups, $\mathbb{Z}(L) \neq 0$ implies that $\mathcal{I}(L) \neq 0$.

One might expect the study of the adjoint actions of $U(L)$ and of $K[G]$ to be somewhat similar because of the common Hopf algebra root. However, this is apparently not the case and indeed Lie results scem to be considerably more clusive. Nevertheless, since delta methods are now available for $U(L)$, there does exist an appropriate Lie analog of Theorem 1.1. For this, let $\Delta=\left\{\ell \in L \mid \operatorname{dim}_{K}[\ell, L]<\infty\right\}$ and let $\Delta_{L}$ denote the subspace of $L$ generated by all finite-dimensional Lie ideals of $L$. Then $\Delta_{L} \subseteq \Delta$ are characteristic Lie ideals of $L$ and we have 
Theorem 2.1. If $L$ is a Lie algebra over a field $K$ of characteristic 0 , then

$$
\mathcal{I}(L)=\left(\mathcal{I}(L) \cap U\left(\Delta_{L}\right)\right) U(L)=U(L)\left(\mathcal{I}(L) \cap U\left(\Delta_{L}\right)\right) .
$$

In particular, if $\Delta_{L}=0$ then $\mathcal{I}(L)=0$.

Proof: We follow the notation of [BP2]. Let $\alpha \in \mathcal{I}(L)$, choose a complementary basis $X$ for $\Delta_{L}$ in $L$ and use it to write $\alpha=\sum_{\mu} \mu \alpha_{\mu}$ based on $\Delta_{L}$. Thus each $\alpha_{\mu} \in U\left(\Delta_{L}\right)$ and each $\mu$ is a distinct straightened monomial in $X$. Our goal is to show that $\alpha_{\mu} \in \mathcal{I}(L)$ for all $\mu$.

It is convenient to use the Hopf comultiplication notation. For example, since $\mu$ is a monomial in $X$, we can write the comultiplication of $\mu$ as $\sum_{(\mu)} \mu_{1} \otimes \mu_{2}$ where the sum contains all ordered pairs $\left\{\mu_{1}, \mu_{2}\right\}$ which are complementary partial products of $\mu$. Since $\mu$ is a straightened monomial in $X$, so also are $\mu_{1}$ and $\mu_{2}$. Furthermore, since $\alpha_{\mu} \in U\left(\Delta_{L}\right)$, the comultiplication of $\alpha_{k}$ is contained in $U\left(\Delta_{L}\right) \otimes U\left(\Delta_{L}\right)$. Therefore we can assume that the sum $\sum_{\left(\alpha_{k}\right)}\left(\alpha_{\mu}\right)_{1} \otimes\left(\alpha_{\mu}\right)_{2}$ involves only terms from $U\left(\Delta_{L}\right)$. We use both these assumptions when we write the comultiplication of $\mu \alpha_{\mu}$ as

$$
\sum_{\left(\mu<\alpha_{\mu}\right)}\left(\mu \alpha_{\mu}\right)_{1} \otimes\left(\mu \alpha_{\mu}\right)_{2}=\sum_{(\mu)} \sum_{\left(\alpha_{\mu}\right)} \mu_{1}\left(\alpha_{\mu}\right)_{1} \otimes \mu_{2}\left(\alpha_{\mu}\right)_{2}
$$

Now $\alpha \in \mathcal{I}(L)$, so for all $r \in U(L)$ we have

$$
\begin{aligned}
0 & =\alpha \cdot r=\sum_{\mu}\left(\mu \alpha_{\mu}\right) \cdot r=\sum_{\mu} \sum_{\left(\mu \alpha_{\mu}\right)}\left(\mu \alpha_{\mu}\right)_{1} r S\left(\left(\mu \alpha_{\mu}\right)_{2}\right) \\
& =\sum_{\mu} \sum_{(\mu)} \sum_{\left(\alpha_{\mu}\right)} \mu_{1}\left(\alpha_{\mu}\right)_{1} r S\left(\mu 2\left(\alpha_{\mu}\right)_{2}\right)
\end{aligned}
$$

and hence

$$
0=\sum_{j} \sum_{(\mu)} \sum_{\left(\alpha_{\mu}\right)} \mu_{1}\left(\alpha_{\mu}\right)_{1} r S\left(\left(\alpha_{\mu}\right)_{2}\right) S\left(\mu_{2}\right) \quad \text { for all } r \in U(L)
$$

Notice that the latter formula is a linear identity in $U(L)$. Furthermore, each $\left(\alpha_{\mu}\right)_{1}$ is contained in $U\left(\Delta_{L}\right)$ and each $\mu_{1}$ is a straightened monomial in $X$. Thus, for any monomial $\sigma$, [BP2, Theorem 4.5(i)] implies that

$$
0=\sum_{\mu} \sum_{(\mu)}^{\prime} \sum_{\left(\alpha_{\mu^{\prime}}\right)}\left(\alpha_{\mu}\right)_{1} r S\left(\left(\alpha_{\mu}\right)_{2}\right) S\left(\mu_{2}\right)
$$


where $\sum_{(\mu)}^{\prime}$ indicates the partial sum of those terms with $\mu_{1}=\sigma$. In particular, if we take $\sigma=1$, then $\mu_{1}=1$ implies that $\mu_{2}=\mu$ and the preceding displayed equation becomes

$$
0=\sum_{\mu} \sum_{\left(\alpha_{\mu}\right)}\left(\alpha_{\mu 2}\right)_{1} r S\left(\left(\alpha_{\mu}\right)_{2}\right) S(\mu) \quad \text { for all } r \in U(L) .
$$

Again, the latter formula is a linear identity in $U(L)$ and this time we observe that $S\left(\left(\alpha_{\mu}\right)_{2}\right) \in U\left(\Delta_{L}\right)$ and that $S(\mu)= \pm \mu$ is plus or minus a straightened monomial in $X$. Thus, for any monomial $\tau$, [BP2, Theorem 4.5(ii)] yields

$$
0=\sum_{\left(\alpha_{\tau}\right)}\left(\alpha_{\tau}\right)_{1} r S\left(\left(\alpha_{\tau}\right)_{2}\right)=\alpha_{\tau} \cdot \tau \quad \text { for all } r \in U(L)
$$

In other words, $\alpha_{\tau} \in \mathcal{I}(L) \cap U\left(\Delta_{L}\right)$ and, since this holds for all such $\tau$, we have

$$
\alpha=\sum_{\tau} \tau \alpha_{\tau} \in U(L)\left(\mathcal{I}(L) \cap U\left(\Delta_{L}\right)\right) .
$$

Thus, since $\mathcal{I}(L) \cap U\left(\Delta_{L}\right)$ is an $L$-stable ideal of $U\left(\Delta_{L}\right)$, we conclude that

$$
\mathcal{I}(L)=U(L)\left(\mathcal{I}(L) \cap U\left(\Delta_{L}\right)\right)=\left(\mathcal{I}(L) \cap U\left(\Delta_{L}\right)\right) U(L)
$$

and the result follows. Furthermore, if $\Delta_{L}=0$, then clearly we have $\mathcal{I}(L) \cap U\left(\Delta_{L}\right)=\mathcal{I}(L) \cap K=0$ and therefore $\mathcal{I}(L)=0$ as required.

The corresponding result in characteristic $p>0$ is decidedly false. For example, let $L=A \times B$ where $A=\left\langle a_{1}, a_{2}, \ldots\right\rangle$ is an infinite-dimensional abelian ideal, $B=K b$ is a onc-dimensional complement and $\left[a_{i}, b\right]=a_{i+1}$ for all $i$. Then it follows exsily that $\Delta_{L}=0$ and that $a^{p} \in \mathbb{Z}(U(L))$ for all $a \in A$. Thus $0=\operatorname{arl} a^{p}=(\operatorname{acl} a)^{p}$, so $a^{p} \in \mathcal{I}(L)$ and therefore $\mathcal{I}(L) \neq 0$.

As is well known, such exarriples occur becanse; in claracteristic $p>0$, ordinary enveloping algebras do not adequately refect their Hopf algebra structure. Indeed, in this concext, one knows that it is more appropriate to consider restricted Lie algebras. Furthermore, if $L$ is any Lic algebra, then there exists a restricted Lie algebra $\hat{L}$ with $U(L)=u(\hat{L})$. Thus, in positive characteristies, the restricted case is really the general case.

If $L$ is a restricted Lie algebri over a field $K$ of characteristic $p>0$, then we let $u(L)$ denote its restricted enveloping algebra. The formulas for the comultiplication, antipode and left adjoint action are of course the same as in the ordinary case and we write

$$
\mathcal{I}(L)=\mathcal{I}(u(L))=\{\alpha \in u(L) \mid \alpha \cdot u(L)=0\}
$$


for the kemel of the adjoint representation. Again $\Delta_{L}$ derotes the subalgebra of $L$ generated by all finite-dimensional (not necessarily restricted) Lie ideals and we let $\Delta$ be as before.

Now delta methods exist for $u(L)$, but unfortunately their statements in the literature are not quite what we require. Nevertheless, the necessary results are true, follow fairly easy from what is known and will be published at some later time. For now we just make two quick observations. First, it is an easy exercise to show that $\Delta_{L}=\Delta$ in the restricted case and, in particular, we see that $\Delta_{L}$ is a characteristic restricted ideal of $L$. Second, the argument of [BP2, Proposition 4.2] applies equally well to restricted enveloping algebras and yields the necessary sharpening of [BP1, Theorem 5.1]. With this, the exact, restricted analog of [BP2, Theorem 4.5] holds and therefore a direct application of the preceding proof yields

Theorem 2.2. If $L$ is a restricted Lie olgebra over a field $K$ of characteristic $p>0$, then

$$
\mathcal{I}(L)=\left(\mathcal{I}(L) \cap u\left(\Delta_{L}\right)\right) u(L)=u(L)\left(\mathcal{I}(L) \cap u\left(\Delta_{L}\right)\right) .
$$

In particular; if $\Delta_{L}=0$ then $I(L)=0$.

The similarity of the preceding two theorems suggests that we should modify our notation somewhat. Thus, for the remainder of this section, we will observe the following conventions.

(1) If $K$ has characteristic 0 , then $L$ is an ordinary Lie algebra and $U(L)$ is, as usual, its ordinary enveloping algebra.

(2) If $K$ has characteristic $p>0$, then $L$ will be a restricted Lie algebra and we change notation to let $U(L)$ denote its restricted enveloping algebra.

As will be apparent, this change allows us to avoid umecessary repetitions.

Next, we also modify our direction somewhat. We know that $L$ is a submodule of $U(L)$ under the adjoint action and, for many reasons, this is a more interesting module structure to study. Thus we will be concerned with faithfulness in this context and we let

$$
\mathcal{J}(L)=\{\alpha \in U(L) \mid \alpha \cdot L=0\}
$$

denote the ideal of $U(L)$ which is the kernel of the action of $U(L)$ on $L$. Obviously $\mathcal{J}(L) \supseteq \mathcal{I}(L)$ and $\mathcal{J}(L) \neq 0$ when $L$ is abelian.

If $L$ is a firite-dimensional Lie algebra, then it is well known that $\mathcal{J}(L) \neq 0$. Indeed, in characteristic 0 , every $0 \neq \ell \in L$ is transcendental 
as an element of $U(L)$, but of course ad $\ell$ is an algebraic linear transformation on the finite-dinensional space $L$. On the other hand, in characteristic $p>0$, the dimension of $U(L)$ is just too large. To be precise, if $\operatorname{din}_{K} L=n \geq 2$, then we have $\operatorname{dim}_{K} U(L)=p^{n} \geq n^{2}=\operatorname{dim}_{K}$ End $_{K}(L)$. Furthermore, equality can only occur when $p=n=2$ and this case is then easily checked by hand. As we will see in the next two lemmas, $\mathcal{J}(L) \neq 0$ seems to be the rule even in infinite-dirnensional situations. Nevertheless, in the final result of this section we will apply delta methods to show that there is an abundant, supply of Lie algebras $L$ with $\mathcal{J}(L)=0$.

Lemma 2.3. Let $A$ be an associative algebra over the field $K$ and let $L$ be a $K$-subspace of $A$ closed under the Lie bracket $[x, y]=x y-y x$. Furtherrnore, if $K$ has characteristic $p>0$, assume that $L$ is closed under taking pth powers. Then $\mathcal{J}(L) \neq 0$ if either

i. $K$ has characteristic 0 and $L$ contains a nonzero algebraic element of $A$, or

ii. $K$ has characteristic $p \geq 5$ and $L$ contains a nonzero idempotent of $A$ or a nonzero element of $A$ of square 0 .

Proof: Notice that $L$ acts on $A$ via $\ell \cdot a=(\operatorname{ad} \ell) a=[\ell, a]$ for all $\ell \in L, a \in A$ and hence the adjoint, action of $U(L)$ on $L$ extends to an action on $A$. Furthermore, we have ad $\ell=\mathcal{L}_{\ell}-\mathcal{R}_{\ell}$ where $\mathcal{L}_{\ell}: A \rightarrow A$ denotes left multiplication by $\ell$ and where $\mathcal{R}_{\ell}: A \rightarrow A$ denotes right multiplication. Of course $\mathcal{L}_{\ell}$ and $\mathcal{R}_{\ell}$ commute as operators.

Now if $0 \neq \ell \in L$ is an algebraic element of $A$, then clearly $\mathcal{L}_{\ell}$ and $\mathcal{R}_{\ell}$ are ajgebraic operators on $A$ and, since they commute, so is ad $\ell=\mathcal{L}_{\ell}-$ $R_{\ell}$. Thus the result follows in characteristic 0 since $\ell$ is transcendental as an element of $U(L)$.

Finally, if $\ell$ is an idempotent element of $A$, then $\mathcal{L}_{\ell}$ and $\mathcal{R}_{\ell}$ are commuting idempotent operators and it follows easily that $(\operatorname{ad} \ell)^{3}=\operatorname{ad} \ell$. Similarly, if $\ell$ is an element of $A$ of square 0 , then $\mathcal{L}_{\ell}^{2}=\mathcal{R}_{\ell}^{2}=0$ and hence $(\operatorname{ad} \ell)^{3}=0$. In either case, ad $\ell$ is algebraic of degree $\leq 3$. On the other hand, if $K$ has characteristic $p>0$ and if $\ell \neq 0$, then $1, \ell, \ell^{2}, \ldots, \ell^{p-1}$ are $K$-linearly independent when viewed as elements of $U(L)$. Thus, if $p \geq 5$, we conclude that $U(L)$ is not faithful on $L$.

In other words, the usual constructions for infinite-dimensional Lie algebras lead to situations which are not faithful. One such construction of particular interest is as follows. If $W$ is a subspace of the vector space $V$, we let

$$
\operatorname{End}_{K}(V ; W)=\left\{t \in \text { End }_{K}(V) \mid t(V) \subseteq W\right\}
$$


Then End $(V ; W)$ is a subalgebra (without 1$)$ of End( $V$ ) and, in particular, it gives rise to a (restricted) Lie algebra which we denote by $\operatorname{gl}(V ; W)$.

Lemma 2.4. Let $L=\operatorname{gl}(V ; W)$ with $W$ an infinite-dimensional proper subspace of $V$. Then $\Delta=0$, but $\mathcal{J}(L) \neq 0$.

Proof: We can easily see that $\Delta=0$ by looking at matrices, but a simple direct, proof is as follows. Let $t$ be a nonzero element of $\mathcal{E}=$ End $(V ; W)$. Since $t \neq 0$ and $V \neq W$, it follows that $t$ does not vanish on $V \backslash W$. Thus we can choose $v \in V \backslash W$ with $t(v)=w \neq 0$ and we note that $\{v, w\}$ is linearly independent since $w \in W$. Now let $\mathcal{S}$ be the subalgebra (without 1) of $\mathcal{E}$ consisting of all $s \in \mathcal{E}$ with $s(v)=0$. Then the independence of $\{v, w\}$ casily implies that the evaluation map $\xi: \mathcal{S} \rightarrow W$ given by $s \mapsto s(w)$ is a $K$-linear epimorphism and therefore the kernel of $\xi$ has infinite codimension in $\mathcal{S}$. Furthermore, if $s \in \mathbb{C}_{S}(t)$, then $s(w)=s t(v)=t s(v)=0$ and thus $\mathbb{C}_{S}(t) \subseteq \operatorname{Ker}(\xi)$. Putting this all together, we see that $\mathbb{C}_{S}(t)$ has infinite codimension in $\mathcal{S}$, so $\mathbb{C}_{\mathcal{E}}(t)$ has infinite codimension in $\mathcal{E}=\operatorname{End}(V, W)$ and this, in turn, implies that $\Delta=0$.

For the second part: we note that $\mathcal{J}(L) \neq 0$ follows immediately from the previous lemma except when $K$ has characteristic 2 or 3 . However, we can offer a simple direct proof which applies to all characteristics. To start with, since $V \neq W$ and $\operatorname{dim}_{K} W \geq 2$, it follows that there exist two linearly independent transformations $x, y \in \operatorname{End}(V ; W)$ with $x(W)=y(W)=0$. The latter conditions then imply that $x \operatorname{End}(V ; W)=$ $y \operatorname{End}(V ; W)=0$ and hence, for all $t \in \operatorname{End}(V ; W)$, we have $[x, t]=-t x$ and $[y, t]=-t y$. Thus $(\operatorname{ard} x)(\operatorname{ad} y) t=t y x=0$ and we conclude that $x y \in \mathcal{J}(L)$. On the other hand, $\{x, y\}$ is linearly inclependent, so $x y \neq 0$ in $U(L)$ and therefore $\mathcal{J}(L) \neq 0$ as required.

Thus we sec that $\Delta=0$ does not imply that $\mathcal{J}(L)$ vanishes and therefore the natural analog of Theorems 2.1 and 2.2 cannot hold in this context.

For any Lie algebra $L$, let $\omega(L)$ denote the augmentation ideal of $U(L)$, namely the ideal of $U(L)$ generated by $L$. Of course, $\omega(L)$ is also the kernel of the algebra epimorphism $U(L) \rightarrow K$ determined by $L \rightarrow 0$ and hence $U(L)=K+\omega(L)$.

Lemma 2.5. Let $L$ be a Lie algebra over the field $K$.

i. There exists a Lie algebra $M \neq 0$ containing $L$ with $\Delta(M)=0$.

ii. If $\Delta(L)=0$, then there exists a Lie algebra $N$ containing $L$ with $\Delta(N)=0$ and $\mathcal{J}(N) \cap U(L)=0$. 
Proof: (i) We can assume that $L$ is an infinite-dimensional Lie algebra and therefore that $\operatorname{dim}_{K} U(L)=\infty$. Now lct $V=U(L)$ and set $W=$ $\omega(L)$. Then, via the left regular representation of $U(L)$, we see that $\omega(L)$ embeds in $\operatorname{End}_{K}(V: W)$. Thus $L \subseteq \operatorname{gl}(V: W)=M$ and the preceding lemma yields the result.

(ii) We are given $\Delta(L)=0$ and we can clearly assume that $L \neq 0$ so that $\operatorname{dim}_{K} L=\infty$. If $N=\omega(L)$ then, via the usual Lie bracket $[x, y]=x y-y x$, it follows thint $N$ is a (restricted) Lie algebra contaning $L$. The goal is to show that $\Delta(N)=0$ and that $\mathcal{J}(N) \cap U(L)=0$.

To start with, if $x \in \Delta(N)$, then cerlainly $x$ is an element of $U(L)$ satisfying $\operatorname{dim}_{K} L / \mathbb{C}_{L}(x)<\infty$. [BP1, Lemma 7.1] therefore implies that $x \in U(\Delta)=K$ and hence $x \in K \cap \omega(L)=0$ as required.

Next, consider the adjoint action of $U(N)$ on $N$ and notice that $L$ acts on $N$ via $\ell \bullet \alpha=[\ell, \alpha]=\ell \alpha-\alpha \ell$ for sall $\ell \in L$ and $\alpha \in N=\omega(L)$. Since the action of $U(L)$ or $N$ is the unique algebra extension of this; it follows that the action of $U(L)$ is identical to the adjoint action of $U(L)$ on $\omega(L)$. Therefore, we have

$$
\mathcal{J}(N) \cap U(L)=\{\beta \in U(L) \mid \beta \cdot \omega(L)=0\}
$$

and, for convenience, we denote the latiter ideal by $\mathcal{I}^{\prime}(L)$. Now it is clear that $\{\beta \in U(L) \mid \beta \cdot K=0\}=\omega(L)$ and therefore $U(L)=K+\omega(L)$ implies that $\omega(L) \cap \mathcal{I}^{\prime}(L)=\mathcal{I}(L)$. Furthermore, $\Delta(L)=0$ so $\mathcal{I}(L)=0$ by Theorcus 2.1 and 2.2 . Thus $\mathcal{I}^{\prime}(L) \subseteq 1$.ann $\omega(L)=0$, since $L$ is infinite dimensional, and the result follows.

lt is now a simple matter to prove

Theorem 2.6. If $L$ is a Lie algebro in charucteristic 0 or a restricted Lie algebra in characteristic $p>0$, then there exists a (restricted) Lie algebra $\tilde{L} \supseteq L$ such that $\Delta(\tilde{L})=0$ and $\mathcal{J}(\tilde{L})=0$.

Proof: According to [emma 2.5(i), we can embed $L$ in a Lie algebra $L_{1}$ with $\Delta\left(L_{1}\right)=0$. Furthermore, starting with $L_{1}$; we can then use Lemma $2.5($ ii $)$ to inductively construct a chain $L_{1} \subseteq L_{2} \subseteq \cdots$ of Lie algebras satisfying $\Delta\left(L_{n_{n}}\right)=0$ and $\mathcal{J}\left(L_{n+1}\right) \cap U\left(L_{n}\right)=0$ for all $n \geq$ 1. Finally, we set $\tilde{L}=\bigcup_{1}^{\infty} L_{n}$ so that $\tilde{L}$ is a (restricted) Lie algebra containing $L$. The gon is to show that $\Delta(\tilde{L})=0$ and that $\mathcal{J}(\tilde{L})=0$. Both of these are quite simple.

First; suppose that $x \in \Delta(\bar{L})$. Then $x \in L_{n}$ for sone $n \geq 1$ and therefore $x \in \Delta(\tilde{L}) \cap L_{n} \subseteq \Delta\left(L_{n}\right)=0$ as required. Next, suppose that $\alpha \in \mathcal{J}(\tilde{L})$. Since $U(\bar{L})$ is cleurly equal to $\bigcup_{1}^{\infty} U\left(L_{\gamma_{r}}\right)$, it follows that $\alpha \in U\left(L_{n}\right)$ for some $n \geq 1$. Furthermore, note that the ad action of 
$L_{n}$ on $U\left(L_{n+1}\right)$ is the restriction of its ad action on $U(\tilde{L})$. Hence the adjoint action of $U\left(L_{n}\right)$ on $U\left(L_{n+1}\right)$ is the restriction of its adjoint action on $U(\tilde{L})$. Thus, since $\alpha \in \mathcal{J}(\tilde{L})$, we have $\alpha \in \mathcal{J}\left(L_{n \div 1}\right) \cap U\left(L_{n}\right)=0$ and the theorem is proved.

A concrete example of a Ijie algebra $L$ with $\mathcal{J}(L)=0$ is the Viresoro algebra in characteristic $0([\mathbf{D}])$.

\section{References}

[BP1] J. BERGEN AND D. S. PASSMAN, Delta methods in enveloping rings, J. Algebra 133 (1990), 277-312.

[BP2] J. BERgEN AND D. S. PASSMAN, Delta methods in enveloping rings II, J. Algebra (to appear).

[D] C. A. DEAN, Private communication.

[I] I. M. IsAaCs, "Character Theory of Finite Groups," Academic Press, New York, 1976.

[P] D. S. PASSMan, "The Algebraic Shucture of Group Rings," Wiley-Interscience, New York, 1977.

[R.S] J. B. Rosser AND L. SCHOEnFEl D, Approximate formulas for some functions of prime numbers, Ilinoois. J. Math. 6 (1962), 64-94.

\footnotetext{
Department of Mathematics

University of Wisconsin

Madison, Wisconsin 53706

U.S.A.
}

Rebut el 29 d'Octubre de 1991 